\title{
Spin dephasing of doped electrons in charge-tunable InP quantum dots: Hanle-effect measurements
}

\author{
Yasuaki Masumoto,* Shuhei Oguchi, Bipul Pal, and Michio Ikezawa \\ Institute of Physics and Tsukuba Advanced Research Alliance (TARA), University of Tsukuba, Tsukuba, Ibaraki 305-8571, Japan
}

(Received 8 September 2006; revised manuscript received 13 October 2006; published 28 November 2006)

\begin{abstract}
The spin dephasing relaxation in single-electron-doped InP quantum dots was studied by means of Hanle measurements. When an InP quantum dot is doped with one electron on average, a narrow Lorentzian dip with half-width of $4.6 \mathrm{mT}$ appeared and was superposed on two Lorentzians with half-widths of 1.54 and $128 \mathrm{mT}$ in the Hanle curve. The half-widths $1.54 \mathrm{~T}, 128 \mathrm{mT}$, and $4.6 \mathrm{mT}$ are ascribed to spin-dephasing relaxation of holes, electron-hole pairs, and doped electrons consistuting negative trions, respectively. The corresponding spin coherence time of the doped electrons at $5 \mathrm{~K}$ is $1.7 \mathrm{~ns}$, which is determined by the frozen fluctuation of nuclear spins in the quantum dots. With increase of temperature, the spin-dephasing rate of the doped electrons increases.
\end{abstract}

DOI: 10.1103/PhysRevB.74.205332

PACS number(s): 78.67.Hc, 71.35.Pq, 72.25.Fe

\section{INTRODUCTION}

Study of semiconductor spins by means of optical orientation has attracted considerable attention of researchers in recent years. Further advance in this field might create quantum information processing devices and spin-electronic (spintronic) devices. ${ }^{1,2}$ The spin relaxation in bulk semiconductors and quantum structures has been extensively studied theoretically and experimentally. ${ }^{3}$ Among these structures, quantum dots (QDs) are promising candidates for quantum spin memory, ${ }^{4}$ because spin relaxation processes are greatly suppressed by the three-dimensional quantum confinement of carriers in QDs. ${ }^{5,6}$ As a result, spin relaxation times in QDs could be long compared to those in bulk semiconductors or two-dimensional structures. Experimental demonstration of long spin relaxation in QDs has been made. The spin relaxation time of excitons is beyond the recombination time at low temperature in InAs, CdSe, and CdTe QDs, as observed by time-resolved photoluminescence ${ }^{7,8}$ (PL) or static circularly polarized PL under a magnetic field. ${ }^{9}{ }^{910}$ In addition, a spin-dephasing time of $3 \mathrm{~ns}$ including the inhomogeneous effect $\left(T_{2}^{*}\right)$ of electrons in chemically synthesized CdSe QDs has been observed at $0 \mathrm{~T}$ and $6 \mathrm{~K}$ by means of a time-resolved Faraday rotation measurement. ${ }^{11}$

In a neutral quantum dot, the spin relaxation time of an optically created electron or hole is restricted by the recombination lifetime. In contrast, this limitation is removed in charged QDs, where a doped electron or a doped hole is present permanently. If optical orientation, long-time storage, and optical readout of the spins of doped electrons or holes can be done, their spins in QDs become leading candidates for a quantum bit. In the case of charge-tunable QDs particularly, we can control the charge state in the QDs with an applied electric bias. ${ }^{12,13}$ From this point of view, the spins of doped electrons or holes in the charged QDs are an attractive research target. The long spin relaxation time $T_{1}$ of doped electrons and holes has been reported in several publications. ${ }^{14-22}$

The spin-dephasing relaxation time $T_{2}^{*}$ is different from the spin relaxation time $T_{1}$. A long spin-dephasing time $T_{2}^{*}$, which is the lifetime of coherent superposition of spin-up and spin-down states, is absolutely imperative for the implementation of spin-based quantum computing and storage. Although a few studies have been made on spin-dephasing relaxation in charged QDs, ${ }^{23-26}$ studies about the mechanisms of spin dephasing are still at the elementary stage.

In this paper, we present our study of the spin-dephasing relaxation of doped electrons in charge-tunable InP QDs by means of the Hanle effect. The Hanle effect measures the dynamical spin orientation of carriers in time units of the Larmor spin precession period. ${ }^{27}$ Although the Hanle measurement is static, it has high sensitivity in measuring the spin-dephasing time of carriers in QDs. ${ }^{23,24,28}$ We succeeded in measuring the Hanle effect of charge-tunable InP QDs with high sensitivity and accuracy of the circular polarization $(0.1 \%)$. We observed optical orientation of the spin of the doped electrons under quasiresonant excitation and measured a spin-dephasing time $T_{2}^{*}$ of $1.7 \mathrm{~ns}$ at $5 \mathrm{~K}$, exceeding the recombination lifetime of $250 \mathrm{ps}$. This spin-dephasing relaxation mechanism is attributed to the frozen fluctuation of nuclear spins in the QDs. We also investigated the spindephasing relaxation mechanism through the temperature dependence of the Hanle effect.

\section{EXPERIMENTAL PROCEDURES}

The sample studied was charge-tunable InP selfassembled QDs grown by gas-source molecular beam epitaxy on an $n^{+}$-type GaAs substrate. In this sample we found submillisecond spin memory. ${ }^{16,17}$ A single layer of InP QDs with a nominal thickness of 4 monolayers was grown between the $100 \mathrm{~nm} \operatorname{In}_{0.5} \mathrm{Ga}_{0.5} \mathrm{P}$ barrier layers. The average lateral diameter of the QDs is $40 \mathrm{~nm}$, and their height is $5 \mathrm{~nm}$. The areal density of the QDs was about $10^{10} \mathrm{~cm}^{-2}$. Under above-barrier excitation, the sample shows a PL band peaked at $1.728 \mathrm{eV}$, as shown in the inset of Fig. 1.

In order to apply an external electric bias, the sample was provided with a transparent indium tin oxide Schottky contact on the top surface and a Au:Ge Ohmic contact on the back surface. Because the lowest electron level in the QDs is close to the Fermi level of the doped substrate, the external electric bias allowed us to control the charged state of the 


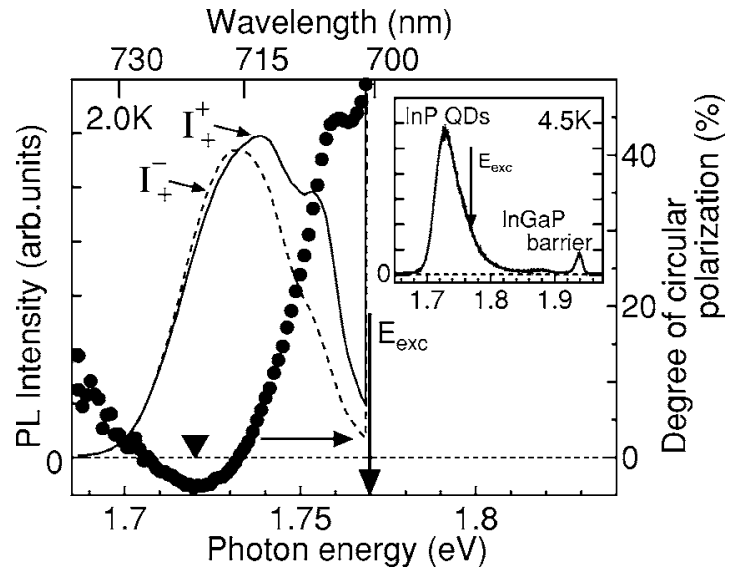

FIG. 1. Circularly polarized photoluminescence spectra of InP quantum dots at $2.0 \mathrm{~K}$ under selective circularly polarized excitation of the dots at $1.771 \mathrm{eV}$, below the $\mathrm{In}_{0.5} \mathrm{Ga}_{0.5} \mathrm{P}$ barrier band gap. The electric bias applied is $-0.1 \mathrm{~V}$. Degree of circular polarization of PL is shown by solid circles. The shoulder seen at the excitationdetection energy difference of $\Delta E=10 \mathrm{meV}$ can be ascribed to the transverse acoustic phonon structure. In the inset, the PL spectrum of the sample under green laser excitation at $2.33 \mathrm{eV}$ is shown and the excitation photon energy of $1.771 \mathrm{eV}$ for the circularly polarized PL experiment is indicated by an arrow.

QDs in the sample. ${ }^{13}$ As is decribed later, a trionic quantum beat measurement showed that there is, on average, one doped electron per dot under a bias of $U=-0.1 \mathrm{~V} .{ }^{13}$ Under positive bias, QDs doped with more than two electrons become dominant, while neutral QDs become dominant below the electric bias of $U=-0.4 \mathrm{~V}$, where an excitonic quantum beat was clearly observed. ${ }^{29}$

A continous-wave Ti:sapphire laser was used for the quasiresonant excitation of InP QDs in the Hanle measurements. In the Voigt configuration (magnetic field is perpendicular to the optical excitation axis and the sample growth direction), a superconducting magnet $(|B| \leqslant 6 \mathrm{~T})$ was used together with a double monochromator. A photoelastic modulator (PEM) operating at the frequency of $42 \mathrm{kHz}$ was used to modulate the polarization of the excitation beam. The PL polarization was measured in the reflection geometry with a quarter-wave $(\lambda / 4)$ plate and a Glan-Thompson linear polarizer as a circular polarization analyzer, and was detected by a photomultiplier with a GaAs (Cs) photocathode and a two-channnel gated photon counter.

Because the spin relaxation time of the nuclei is much slower than the modulation period of the PEM $(24 \mu \mathrm{s})$, the nuclear spin cannot follow the alternating spin polarization of the electrons and there is no dynamic polarization of the nuclei. ${ }^{27}$ This permitted us to eliminate the effect of the dynamic nuclear polarization on the optical orientation of the electrons. The degree of circular polarization, $\rho$, is defined by

$$
\rho=\frac{I_{+}^{+}-I_{+}^{-}}{I_{+}^{+}+I_{+}^{-}},
$$

where $I_{+}^{+}$and $I_{+}^{-}$are the intensities of the $\sigma^{+}$PL component under $\sigma^{+}$and $\sigma^{-}$pumping, respectively. In a Hanle measure- ment, $\rho$ is measured as a function of the transverse magnetic field.

\section{EXPERIMENTAL RESULTS AND DISCUSSION}

\section{A. Negative circular polarization of luminescence in single- electron-doped quantum dots}

The circularly polarized PL spectra of InP QDs under the electric bias of $U=-0.1 \mathrm{~V}$ at $2 \mathrm{~K}$ are shown in Fig. 1. The excitation photon energy $E_{\text {exc }}=1.771 \mathrm{eV}$ excites the QDs quasiresonantly. The degree of circular polarization of the PL is more than $40 \%$ within the excitation-detection energy difference $(\Delta E)$ of $10 \mathrm{meV}$ and decreases with increase of $\Delta E$. The degree of circular polarization is positive above $1.733 \mathrm{eV}$ and changes its sign on increasing $\Delta E$ further. Negative circular polarization (NCP) was observed between $\Delta E$ of 39 and $63 \mathrm{meV}$. The NCP became maximum at $\Delta E$ of about $49 \mathrm{meV}$. There is one doped electron per dot on average under the electric bias $U=-0.1 \mathrm{~V}$, which is indispensable for NCP. In fact, NCP was observed only in the narrow range of the electric bias $-0.4 \leqslant U \leqslant 0.1 \mathrm{~V}$. NCP was not observed when the excitation photon energy $E_{\text {exc }}=1.746 \mathrm{eV}$ is closer to the detection photon energy $E_{\mathrm{det}}=1.722 \mathrm{eV}$. Then the degree of circular polarization of the PL is positive at the PL band and monotonically decreases with increase of $\Delta E$. These observations show that spin relaxation of photocreated electrons and holes takes place in part in a phonon-mediated relaxation process. ${ }^{30}$ With increase of $\Delta E$, phonon-mediated relaxation needs more time and spin relaxation proceeds. The successive spin relaxation associated with phonon-mediated relaxation was observed in $\mathrm{CdSe}$ and CdTe QDs. ${ }^{8,31}$ To explain the NCP, however, another spin flip-flop process described below and optical orientation of the doped electrons are needed.

A model for the mechanism of the NCP formation in quasiresonantly excited electron-doped InP QDs is described in Refs. 16 and 17 and is shown in Fig. 2. Here, we explain the mechanism concisely. A quantum dot doped with one electron is either a PQD in which the spin of the doped electron is oriented parallel to the helicity of the circularly polarized excitation or an AQD in which the spin is oriented antiparallel to the helicity. The right circularly $\left(\sigma^{+}\right)$polarized quasiresonant excitation creates electrons with spin $\downarrow$ and holes with spin $\Uparrow$ in the excited state of the QDs for $12 \mu \mathrm{s}$. A hot bright trion composed of a hole $\Uparrow$ and two electrons $\sqrt{1 / 2}(\downarrow \uparrow+\uparrow \downarrow)$ is formed in an AQD [Fig. 2(b)], while a bright trion composed of a hole $\Uparrow$ and two electrons $\downarrow \downarrow$ is formed in a PQD [Fig. 2(a)]. In the $\downarrow \downarrow \Uparrow$ QDs, the photoexcited electrons cannot relax to the ground state without flip of their spins due to Pauli blocking. However, the electronhole pair can go through a flip-flop process in which both the electron spin and the hole spin are flipped simultaneously, and energy relaxation occurs. This flip-flop process is due to the presence of an anisotropic component of the exchange coupling and is considered to explain the NCP. ${ }^{18,32,33}$ After the flip-flop process, electron-hole recombination occurs in the trion ground state $\uparrow \downarrow \Downarrow$ and the sign of the PL polarization inverts $\left(\sigma^{-}\right)$. Consequently, the degree of circular polariza- 


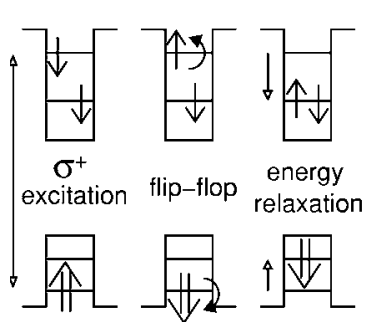

(a) $P Q D$

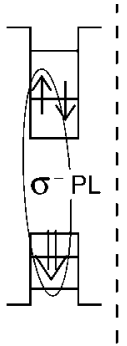

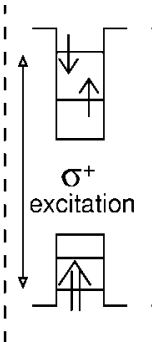

(1)

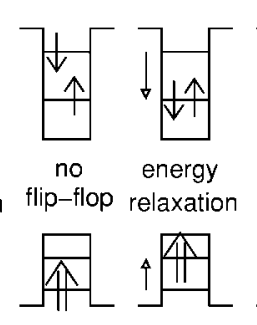

(b) $A Q D$

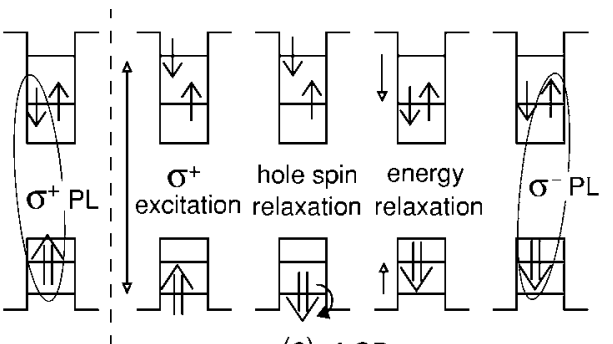

(c) $A Q D$

FIG. 2. Schematic spin orientation of electrons and a hole in a quantum dot, after circularly polarized photoexcitation generates an electron-hole pair at the excited state in a single-electron-doped quantum dot. (a) A process to geneate negative circularly polarized luminescence in a PQD. PQD denotes a quantum dot in which the spin of the doped electron is oriented parallel to the helicity of the circularly polarized excitation. (b) A process to generate positive circularly polarized luminescence in an AQD. AQD denotes a quantum dot in which the spin of the doped electron is oriented antiparallel to the helicity of the circularly polarized excitation. (c) Spin flip of a single particle-hole in an AQD, which causes optical pumping of the doped electron.

tion of the PL should be negative in a PQD. In the case of the AQD, a hot electron-hole pair in the trion excited state $\sqrt{1 / 2}(\downarrow \uparrow+\uparrow \downarrow) \Uparrow$ can relax to the trion ground state $\uparrow \downarrow \Uparrow$ without the flip-flop process because the spins of the photoexcited and doped electrons are antiparallel. Then the electronhole recombination occurs in the ground state and gives positive PL polarization $\left(\sigma^{+}\right)$. Therefore, the degree of circular polarization of the PL should be positive in the AQD. In addition to the process shown in Fig. 2(b), spin flip of a single particle hole is assumed to take place in part of the AQD, because hole spin stability in the AQD is considered to be lower than that in the PQD. This is because the electronhole exchange interaction is reduced for electron pairs having zero total spin. Therefore we consider the spin flip of a single particle hole in an AQD, as is shown in Fig. 2(c). This process aligns the spin of a doped electron and makes the type conversion of AQD to PQD, after the negative circularly polarized PL is emitted. The NCP is explained by considering the simultaneous spin flip-flop process of a photocreated electron-hole pair in a PQD and optical pumping of the doped electrons causing the type conversion of AQD to PQD under circularly polarized excitation. ${ }^{16,17}$ Rise of the NCP observed in the time-resolved circularly polarized luminescence of InP QDs shows the flip-flop time to be about 60 ps. $^{16}$

\section{B. The Hanle curve in single-electron-doped quantum dots}

For the excitation photon energy of $1.771 \mathrm{eV}$ and the detection energy of $1.722 \mathrm{eV}$, the Hanle curves of the singleelectron-doped InP QDs are shown in Fig. 3(a) and the narrowest dip of the lower curve is enlarged and displayed in Fig. 3(b). The lower Hanle curve was well expressed by a sum of three Lorentzians,

$$
\rho(B)=A_{0}+\frac{A_{\mathrm{NCP}}}{1+\left(B / B_{1}\right)^{2}}+\frac{A_{2}}{1+\left(B / B_{2}\right)^{2}}+\frac{A_{3}}{1+\left(B / B_{3}\right)^{2}},
$$

where $B_{i}=\hbar / g_{i} \mu_{\mathrm{B}} T_{2, i}^{*}(i=1,2,3)$ was used in the fitting (solid line). The fitting parameters are $A_{0}=0.10 \%, A_{\mathrm{NCP}}$ $=-1.43 \%, \quad B_{1}=4.6 \mathrm{mT}, \quad A_{2}=-3.97 \%, \quad B_{2}=128 \mathrm{mT}, \quad A_{3}$
$=2.30 \%$, and $B_{3}=1.54 \mathrm{~T}$. Here the three Lorentzians are categorized by the range of magnetic field and called the first Lorentzian $\left(A_{\mathrm{NCP}}, B_{1}\right)$, the second Lorentzian $\left(A_{2}, B_{2}\right)$, and the third Lorentzian $\left(A_{3}, B_{3}\right)$ from small to large magnetic field, respectively. The first negative Lorentzian looks like a sharp dip with half width at half maximum $B_{1}$ of $4.6 \mathrm{mT},{ }^{34}$ from which a scaled spin-dephasing time $g T_{2}^{*}$ of $2.5 \mathrm{~ns}$ is obtained from $g T_{2}^{*}=\hbar / \mu_{\mathrm{B}} B_{1}$. The amplitude $A_{\mathrm{NCP}}$ of the dip increases in proportion to the logarithm of the excitation power density. ${ }^{35}$

The sharp dip in the Hanle curve disappears when the excitation photon energy is $1.746 \mathrm{eV}$ and the detection photon energy is $1.722 \mathrm{eV}$ (excitation-detection energy difference $\Delta E=25 \mathrm{meV}$ ). The Hanle curve consits of two positive Lorentzians. The fitting parameters are $A_{0}=0.89 \%, A_{2}$ $=14.34 \%, B_{2}=82.4 \mathrm{mT}, A_{3}=5.85 \%$, and $B_{3}=1.55 \mathrm{~T}$. The linewidth $B_{2}$ is smaller than that in the Hanle curve for $\Delta E$

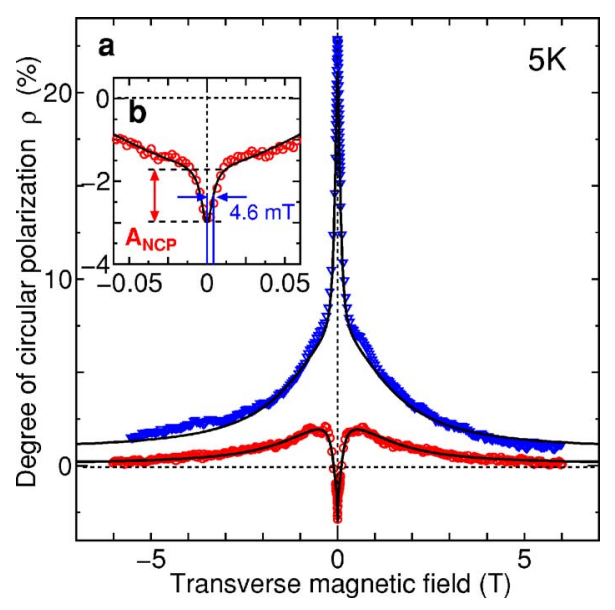

FIG. 3. (Color online) (a) Hanle curves for InP QDs at $5 \mathrm{~K}$ under $-0.1 \mathrm{~V}$. The excitation photon energy was $1.771 \mathrm{eV}$ (circles) or $1.746 \mathrm{eV}$ (triangles) and the detection energy was $1.722 \mathrm{eV}$. A sum of three Lorentzians is used in the fit of the lower Hanle curve, while a sum of two Lorentzians is used in the fit of the upper Hanle curve. The narrowest Lorentzian dip observed in the lower Hanle curve for the excitation at $1.771 \mathrm{eV}$ has half width at half maximum of $4.6 \mathrm{mT}$ as shown in (b). 
$=50 \mathrm{meV}$ by $35 \%$, while the linewidth $B_{3}$ is equal to that in the Hanle curve for $\Delta E=50 \mathrm{meV}$.

We can neglect the spin-dephasing relaxation of an electron in the ground state of negative trions within the electron-hole recombination time, because the ground state of negative trions consist of solely a singlet formed by two electrons $\sqrt{1 / 2}(\uparrow \downarrow-\downarrow \uparrow)$. We assume no hole spin-dephasing relaxation in the ground state (Kramers doublet) of negative trions within the electron-hole recombination time. The assumption is supported by the experimental reports of the high spin stability of holes for CdSe (Ref. 19) and InAs QDs. ${ }^{20}$ Thus the spin-dephasing relaxation observed by the Hanle effect within the electron-hole recombination time is the spin-dephasing relaxation during the energy relaxation of a photocreated electron-hole pair into a trion in the ground state.

The spin state of a photocreated electron-hole pair can change as a result of the spin flip of an electron, a hole, or an exciton. In an exciton, the stability of the hole spin is lower than that of the electron spin, because of the mixing in the valence band. In fact, in the phonon-mediated relaxation in quantum structures, spin relaxation of holes is known to be much faster than spin relaxation of electrons and in the range of picoseconds. ${ }^{36}$ In case the electron-hole exchange energy $\delta$ is larger than $\hbar / \tau_{h}$, where $\tau_{h}$ is the single-particle hole spin-flip time, stability of the hole spin within the exciton is high and spin flip of the exciton becomes the major spin relaxation process. ${ }^{37}$ In the opposite case, single-particle spin relaxation of the electron and the hole becomes predominant. The electron-hole exchange energy $\delta$ in InP QDs is around $140 \mu \mathrm{eV} .{ }^{29,38}$ The corresponding time $\hbar / \delta$ is 4.7 ps. Because $\tau_{h}$ is considered to be comparable with $\hbar / \delta$, we assume that spin-dephasing relaxation of single-particle holes and excitons (electron-hole pairs) takes place simultaneously. The spin flip of the single-particle hole is displayed schematically in Fig. 2(c) and spin flip of excitons (electron-hole pairs) is shown in Fig. 2(a). The amplitude of polarization $\left|A_{3}\right|$ is smaller than $\left|A_{2}\right|$ and $B_{3}$ is larger than $B_{2}$ by an order of magnitude, as is seen from two Hanle curves in Fig. 3. This leads to the assignment that the scaled spin-dephasing time $g T_{2}^{*}=\hbar / \mu_{\mathrm{B}} B_{3}$ of $7.3 \mathrm{ps}$ is the spin-dephasing time of holes. The $g$ factor of a heavy hole is $g_{\mathrm{h} \perp}=0.25$ around $1 \mathrm{~T}$ under a magnetic field applied in perpendicular to the growth direction. ${ }^{39}$ From $g_{\mathrm{h} \perp}=0.25$, we obtain the spin-dephasing time of the single-particle holes $T_{2}^{*}=29.2 \mathrm{ps}$. Thus, the third Lorentzian represents depolarization by single-particle hole spins under the transverse magnetic field. It is comparable with the phonon-mediated energy relaxation time of electron-hole pairs, $37 \mathrm{ps}$ for an excitation-detection energy difference of $\Delta E=50 \mathrm{meV}$, in InP quantum dots studied by us. ${ }^{40-42}$

In an electron-doped QD, the circular polarization of PL can change its sign only when the spin of a hole is flipped. The negative $A_{2}$ observed for the excitation photon energy of $1.771 \mathrm{eV}$ shows that the spin of a hole is flipped. Based on the model forming the negative circular polarization, an electron is simultaneously flipped in a PQD. When the excitation photon energy is $1.746 \mathrm{eV}, A_{2}$ is positive and $B_{2}$ is smaller than that for the excitation photon energy of $1.771 \mathrm{eV}$ by $35 \%$. This characteristic show that the second Lorentzian

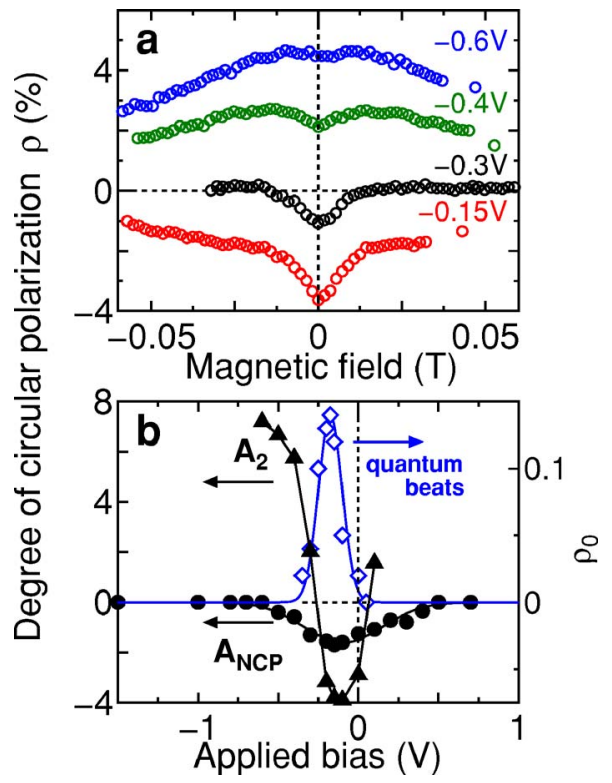

FIG. 4. (Color online) (a) The sharpest Hanle dip vs the electric bias. (b) Closed circles fitted by a Gaussian with full width at half maximum of $\Delta U=0.46 \mathrm{~V}$ show the amplitude of the sharp (first) Lorentzian component. Open diamonds show the amplitude of the measured trionic quantum beat. Triangles show Lorentzian fitting parameter $A_{2}$ for Hanle curves vs the electric bias. Peak (dip) positions of the three fitting lines are in accord.

comes from the spin-dephasing relaxation of excitons (electron-hole pairs), which is the simultaneous spin flip of electron-hole pairs. With the decrease of the excitationdetection energy difference $\Delta E$, spin relaxation becomes more incomplete. The positive $A_{2}$ observed for the excitation photon energy of $1.746 \mathrm{eV}$ means that phonon-mediated energy relaxation of a hole is completed before the spin flip of an electron-hole pair. The scaled spin-dephasing time $g T_{2}^{*}$ of $90 \mathrm{ps}$ comes from the spin flip relaxation of excitons (electron-hole pairs). Photocreated electron-hole pairs precess in a transverse magnetic field and spin-flip relaxation takes place by chance during the precession. With increase of the magnetic field, the precession period becomes shorter than the spin-flip relaxation time and magnetic depolarization takes place for electron-hole pairs. The $g$ factors of an electron and a heavy hole are $g_{e}=1.5$ and $g_{\mathrm{h} \perp}=0.25$, respectively, under a magnetic field applied perpendicular to the growth direction. ${ }^{38,39}$ Therefore the spin-dephasing relaxation times of photoexcited electron-hole pairs are evaluated to be $51 \mathrm{ps}$. The value almost agrees with the simultaneous spin-flip time of an electron-hole pair, $60 \mathrm{ps,} \mathrm{observed} \mathrm{in} \mathrm{the}$ time-resolved circularly polarized luminescence of the same sample. ${ }^{16,43}$ Therefore, we conclude that the second Lorentzian $\left(A_{2}, B_{2}\right)$ comes from the spin-dephasing relaxation of photocreated electron-hole pairs caused by their spin flip.

The sharpest (first) Hanle dips and the dependence of the Lorentzian amplitude parameters describing the Hanle curves on the electric bias are shown in Fig. 4. The amplitude of the sharpest (first) Lorentzian dip has a maximum around $U=-0.15 \mathrm{~V}$ as is seen in Figs. 4(a) and 4(b). Open diamonds show the amplitude $\left(\rho_{0}\right)$ of the measured trionic quantum beat observed under the excitation at $1.771 \mathrm{eV}$ and 
detection at $1.753 \mathrm{eV}$. Note that the two peaks of the trionic quantum beat and the amplitude of the sharpest (first) Lorentzian dip are in accord at $U=-0.15 \mathrm{~V}$. Observation of a trionic quantum beat ensures the presence of a single doped electron in a QD. ${ }^{13}$ Because of the longest spin-dephasing time and the exact coincidence of the sharpest Hanle dip with the trionic quantum beat, the sharpest Lorentzian was assigned to the spin-dephasing relaxation of the doped electron. The half-width of the sharpest Lorentzian $B_{1}=4.6 \mathrm{mT}$ gives the scaled spin-dephasing time $\left(g T_{2}^{*}=2.5 \mathrm{~ns}\right)$ of the doped electrons. From $g_{e}=1.5$, we get the spin-dephasing time $T_{2}^{*}=1.7 \mathrm{~ns}$ for the doped electron. ${ }^{38}$ The recombination lifetime in InP QDs is $250 \mathrm{ps}$ by the time-resolved PL measurement. ${ }^{16}$ The $1.7 \mathrm{~ns}$ spin-dephasing time of the doped electron obtained in our work is much longer than the recombination lifetime.

Figure 4(b) shows the dependence of the second Lorentzian amplitude $A_{2}$ (solid triangles) of the Hanle curve on the electric bias. The second Lorentzian changed its sign drastically under negative bias. Negative $A_{2}$ is observed under electric bias ranging from -0.5 to $+0.0 \mathrm{~V}$, where the trionic quantum beat is observed. The scaled spin-dephasing time $g T_{2}^{*}$ is constant and is about $100 \mathrm{ps}$ under negative electric bias. It is assigned to the spin-flip time of photocreated electron-hole pairs not only in electron-doped QDs but also in neutral QDs. The negative $A_{2}$ shows that the spins of photocreated electrons and holes flip in the presence of a doped electron in a QD and that the number of PQDs exceeds that of AQDs. Strong anisotropic exchange interaction between an electron and a hole photocreated in a QD causes efficient spin flip in both electron-doped QDs and neutral QDs similarly.

\section{Spin dephasing of electrons in doped quantum dots}

Let us consider what spin-dephasing relaxation mechanism is dominant in the spin-dephsing time $T_{2}^{*}=1.7 \mathrm{~ns}$ of doped electrons in QDs. The D'yakonov-Perel' mechanism, the Elliott-Yafet mechanism, and the Bir-Aronov-Pikus mechanism are well known as spin relaxation mechanisms worked dominantly in bulk semiconductors. ${ }^{27}$ These mechanisms are mostly suppressed for the localized electrons in QDs. The dominant dephasing relaxation mechanism of the localized electrons in QDs is the hyperfine interaction with nuclear spins at low temperatures. ${ }^{44-47}$ The localized electrons are influenced by fluctuations in the local nuclear spin environment. The nuclear spins are static during the precession cycle of an electron spin but fluctuate during its many precessions. The fluctuating spins, via the hyperfine interaction, behave like an effective magnetic field and causes the dephasing of the electron spin precession. Merkulov et al. showed that the dephasing time for this process is

$$
T_{\Delta}=\frac{\hbar}{\mu_{\mathrm{B}} g_{\mathrm{e}} \Delta_{\mathrm{B}}}=\hbar\left(n^{2} \sum_{j} I^{j}\left(I^{j}+1\right)\left(A^{j}\right)^{2} /\left(3 N_{\mathrm{L}}\right)\right)^{-1 / 2},
$$

where $\Delta_{\mathrm{B}}$ is the dispersion of the nuclear hyperfine field distribution, $N_{\mathrm{L}}$ the number of nuclei interacting with the electron in the QD, $A^{j}$ the hyperfine constant, $I^{j}$ the spin of the $j$ th nucleus, and $n$ the number of nuclei per unit cell. ${ }^{44,48}$

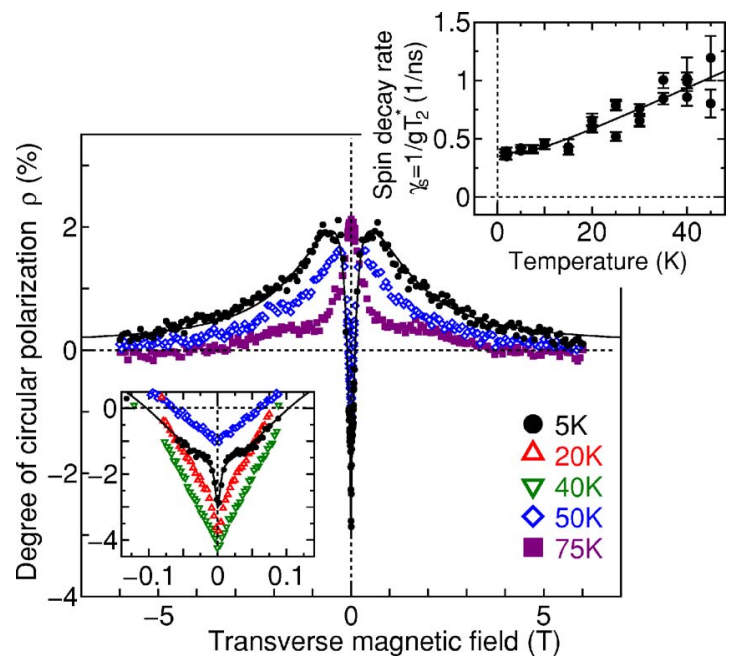

FIG. 5. (Color online) Hanle curve vs temperature under $-0.1 \mathrm{~V}$ bias. The excitation photon energy was $1.771 \mathrm{eV}$ and the detection photon energy was $1.722 \mathrm{eV}$. The lower inset shows that the sharpest first Lorentzian component broadens and fades away at elevated temperature. The upper figure shows the temperature dependence of the spin dephasing rate $\gamma_{\mathrm{s}}=1 / g T_{2}^{*}$ deduced from the sharpest Lorentzian width as a funtion of temperature.

The hyperfine constants of $\operatorname{In}\left(I_{\mathrm{In}}=9 / 2\right)$ and $\mathrm{P}\left(I_{\mathrm{P}}=1 / 2\right)$ are $A_{\text {In }}=56 \mu \mathrm{eV}$ and $A_{\mathrm{P}}=0.5 \mu \mathrm{eV}$, respectively. ${ }^{44,49}$ For an average lens-shaped dot size (base diameter is $40 \mathrm{~nm}$, height is $5 \mathrm{~nm}$ ), we estimate that the number of nuclei in interaction with the electron is $N_{\mathrm{L}} \approx 1.4 \times 10^{5}$. Then Eq. (3) gives $T_{\Delta}$ $\sim 0.8 \mathrm{~ns}$. This is in agreement with the experimental value $T_{2}^{*}=1.7 \mathrm{~ns}$ in order of magnitude and therefore the spindephasing relaxation for the doped electrons in InP QDs can be attributed to the hyperfine interaction with randomly oriented nuclear spins.

Temperature increase modified the Hanle curve of the electron-doped InP QDs, as is shown in Fig. 5. The sharpest (first) Lorentzian dip broadened and faded away at elevated temperatures. The second Lorentzian disappears above $60 \mathrm{~K}$. As a result, the sign at the peak of the Hanle curve was changed from minus to plus at higher temperature. These observations mean the phonon-mediated spin-dephasing relaxation dominates over the spin-flip relaxation above $60 \mathrm{~K}$. The inset of Fig. 5 shows the temperature dependence of the spin dephasing rates $\left(\gamma_{\mathrm{s}}\right)$ for the sharpest (first) Lorentzian. The spin-dephasing time $T_{2}^{*}$ decreases monotonically with increasing temperature. The spin decay rate $\left(1 / T_{1}\right)$ in the same sample, electron-doped InP QDs, increases with increase of temperature, and its temperature dependence shows that the dominant spin relaxation mechanism is the twophonon Orbach process ${ }^{17}$ that is, thermally activated spin relaxation due to the phonon-medicated coupling of the ground and excited electron states. ${ }^{50}$ The spin decay rate is given by $\gamma_{\mathrm{s}} \sim \gamma_{0}+\gamma_{1}\left[\exp \left(E_{\mathrm{a}} / k_{\mathrm{B}} T\right)-1\right]^{-1}\left(E_{\mathrm{a}}\right.$ is the activation energy, $k_{\mathrm{B}}$ the Boltzmann constant, and $\gamma_{0}$ the spin decay rate arising from temperature-independent relaxation mechanisms). The expression was used for fitting with parameters $\gamma_{0}=0.38, \gamma_{1}=0.39$, and $E_{\mathrm{a}}=1.8 \mathrm{meV}$ in the inset of Fig. 5 and good agreement was obtained. Therefore, the two- 
phonon Orbach process dominates over the spin-dephasing relaxation at elevated temperatures similarly to the situation with the spin relaxation $\left(1 / T_{1}\right)$.

We obtained the spin-dephasing time $T_{2}^{*}=1.7 \mathrm{~ns}$ of doped electrons in InP QDs. This time exceeds the recombination lifetime of $250 \mathrm{ps}$ in the InP QDs we studied. ${ }^{16}$ However, it is substantially shorter than the spin memory time $\left(T_{1}\right)$ of doped electrons in charged InP QDs in the microsecond range observed under a longitudinal magnetic field of $0.1 \mathrm{~T} .{ }^{16,17}$ Under a longitudinal magnetic field, the relaxation of electron spins due to the frozen fluctuation of nuclear spins is much supressed, while the dephasing of electron spins is not suppressed in the transverse magnetic field. ${ }^{44,48}$ This is the reason why the spin-dephasing time $T_{2}^{*}$ we observed is much shorter than the spin memory time.

\section{CONCLUSIONS}

The spin-dephasing relaxation in charge-tunable InP quantum dots was studied by means of a Hanle measurement. When an InP quantum dot is doped by one electron on an average, a narrow Lorentzian dip with half-width of $4.6 \mathrm{mT}$ appeared and was superposed on a negative Lorentzian with half-width of $128 \mathrm{mT}$ and a positive Lorentzian with halfwidth of $1.54 \mathrm{~T}$ in the Hanle curve. The half-widths $1.54 \mathrm{~T}$, $128 \mathrm{mT}$, and $4.6 \mathrm{mT}$ are ascribed to the spin-dephasing relaxation of photocreated holes, photocreated electron-hole pairs, and doped electrons consistuting negative trions, respectively. The corresponding spin-dephasing time of the doped electrons at $5 \mathrm{~K}$ is $1.7 \mathrm{~ns}$, which is determined by the frozen fluctuation of nuclear spins in the quantum dots. With increase of temperature, the spin-dephasing rate of the doped electrons increases.

\section{ACKNOWLEDGMENTS}

The authors thank I. V. Ignatiev for many helpful discussions and H.-W. Ren for the sample growth. This work was supported by Grants-in-Aid for Scientific Research No. 13852003 and No. 18204028 from MEXT (Ministry of Education, Science, Sports, and Culture) of Japan and the "R\&D Promotion Scheme Funding International Joint Research" promoted by NICT (National Institute of Information and Communications Technology) of Japan.
*Electronic address: shoichi@ sakura.cc.tsukuba.ac.jp; URL: http:// www.sakura.tsukuba.ac.jp/ masumoto/

${ }^{1}$ S. A. Wolf, D. D. Awschalom, R. A. Buhrman, J. M. Daughton, S. von Molnár, M. L. Roukes, A. Y. Chtchelkanova, and D. M. Treger, Science 294, 1488 (2001).

${ }^{2}$ Semiconductor Spintronics and Quantum Computation, edited by D. D. Awschalom, D. Loss, and N. Samarth (Springer, Berlin, 2002).

${ }^{3}$ I. Zutić, J. Fabian, and S. Das Sarma, Rev. Mod. Phys. 76, 323 (2004).

${ }^{4}$ D. Loss and D. P. DiVincenzo, Phys. Rev. A 57, 120 (1998).

${ }^{5}$ A. V. Khaetskii and Y. V. Nazarov, Phys. Rev. B 61, 12639 (2000).

${ }^{6}$ L. M. Woods, T. L. Reinecke, and Y. Lyanda-Geller, Phys. Rev. B 66, 161318(R) (2002).

${ }^{7}$ M. Paillard, X. Marie, P. Renucci, T. Amand, A. Jbeli, and J. M. Gérard, Phys. Rev. Lett. 86, 1634 (2001).

${ }^{8}$ M. Scheibner, G. Bacher, S. Weber, A. Forchel, Th. Passow, and D. Hommel, Phys. Rev. B 67, 153302 (2003).

${ }^{9}$ S. Mackowski, T. A. Nguyen, H. E. Jackson, L. M. Smith, J. Kossut, and G. Karczewski, Appl. Phys. Lett. 83, 5524 (2003).

${ }^{10}$ S. Mackowski, T. A. Nguyen, T. Gurung, K. Hewaparakrama, H. E. Jackson, L. M. Smith, J. Wrobel, K. Fronc, J. Kossut, and G. Karczewski, Phys. Rev. B 70, 245312 (2004).

${ }^{11}$ J. A. Gupta, D. D. Awschalom, X. Peng, and A. P. Alivisatos, Phys. Rev. B 59, R10421 (1999).

${ }^{12}$ R. J. Warburton, C. Schäflein, D. Haft, F. Bickel, A. Lorke, K. Karrai, J. M. Garcia, W. Schoenfeld, and P. M. Petroff, Nature (London) 405, 926 (2000).

${ }^{13}$ I. E. Kozin, V. G. Davydov, I. V. Ignatiev, A. V. Kavokin, K. V. Kavokin, G. Malpuech, H.-W. Ren, M. Sugisaki, S. Sugou, and Y. Masumoto, Phys. Rev. B 65, 241312(R) (2002).

${ }^{14}$ T. Kawazoe, Y. Masumoto, and T. Mishina, Phys. Rev. B 47,
10452 (1993).

${ }^{15}$ I. V. Ignatiev, T. Okuno, S. Yu. Verbin, I. A. Yugova, and Y. Masumoto, Physica E (Amsterdam) 17, 361 (2003).

${ }^{16}$ M. Ikezawa, B. Pal, Y. Masumoto, I. V. Ignatiev, S. Yu. Verbin, and I. Ya. Gerlovin, Phys. Rev. B 72, 153302 (2005).

${ }^{17}$ B. Pal, M. Ikezawa, Y. Masumoto, and I. V. Ignatiev, J. Phys. Soc. Jpn. 75, 54702 (2006).

${ }^{18}$ S. Cortez, O. Krebs, S. Laurent, M. Senes, X. Marie, P. Voisin, R. Ferreira, G. Bastard, J.-M. Gérard, and T. Amand, Phys. Rev. Lett. 89, 207401 (2002).

${ }^{19}$ T. Flissikowski, I. A. Akimov, A. Hundt, and F. Henneberger, Phys. Rev. B 68, 161309(R) (2003).

${ }^{20}$ S. Laurent, B. Eble, O. Krebs, A. Lemaître, B. Urbaszek, X. Marie, T. Amand, and P. Voisin, Phys. Rev. Lett. 94, 147401 (2005).

${ }^{21}$ R. Hanson, B. Witkamp, L. M. K. Vandersypen, L. H. Willems van Beveren, J. M. Elzerman, and L. P. Kouwenhoven, Phys. Rev. Lett. 91, 196802 (2003).

${ }^{22}$ M. Kroutvar, Y. Ducommun, D. Heiss, M. Bichler, D. Schuh, G. Abstreiter, and J. J. Finley, Nature (London) 432, 81 (2004).

${ }^{23}$ R. I. Dzhioev, B. P. Zakharchenya, V. L. Korenev, P. E. Pak, D. A. Vinokurov, O. V. Kovalenkov, and I. S. Tarasov, Phys. Solid State 40, 1587 (1998).

${ }^{24}$ A. S. Bracker, E. A. Stinaff, D. Gammon, M. E. Ware, J. G. Tischler, A. Shabaev, Al. L. Efros, D. Park, D. Gershoni, V. L. Korenev, and I. A. Merkulov, Phys. Rev. Lett. 94, 047402 (2005).

${ }^{25}$ N. P. Stern, M. Poggio, M. H. Bartl, E. L. Hu, G. D. Stucky, and D. D. Awschalom, Phys. Rev. B 72, 161303(R) (2005).

${ }^{26}$ M. V. Gurudev Dutt, J. Cheng, B. Li, X. Xu, X. Li, P. R. Berman, D. G. Steel, A. S. Bracker, D. Gammon, S. E. Economou, R.-B. Liu, and L. J. Sham, Phys. Rev. Lett. 94, 227403 (2005).

${ }^{27}$ Optical Orientation, edited by F. Meier and B. P. Zakharchenya, 
Modern Problems in Condensed Matter Sciences Vol. 8 (NorthHolland, Amsterdam, 1984).

${ }^{28}$ R. J. Epstein, D. T. Fuchs, W. V. Schoenfeld, P. M. Petroff, and D. D. Awschalom, Appl. Phys. Lett. 78, 733 (2001).

${ }^{29}$ I. A. Yugova, I. Ya. Gerlovin, V. G. Davydov, I. V. Ignatiev, I. E. Kozin, H. W. Ren, M. Sugisaki, S. Sugou, and Y. Masumoto, Phys. Rev. B 66, 235312 (2002).

${ }^{30}$ Single-dot spectroscopy of an InP quantum dot in a similar sample shows that the energy difference between the $s$ shell and the $p$ shell is $17 \mathrm{meV}$ and that between the $s$ shell and the $d$ shell is $35 \mathrm{meV}$ [Y. Masumoto, K. Mizuochi, K. Bando, and Y. Karasuyama, J. Lumin. 122-123, 424 (2007)]. The excitationdetection energy difference $\Delta E=50 \mathrm{meV}$ corresponds to the excitation of the $d$ shell or a higher shell and $\Delta E=25 \mathrm{meV}$ corresponds to the excitation of the $p$ shell. The longitudinal optical (LO) phonon energy of InP is $45 \mathrm{meV}$, so that excess energy of $25 \mathrm{meV}$ needs acoustic-phonon-mediated relaxation and excess energy of $50 \mathrm{meV}$ needs LO- plus acoustic-phononmediated relaxation.

${ }^{31}$ Yu. G. Kusrayev, A. V. Koudinov, B. P. Zakharchenya, S. Lee, J. K. Furdyna, and M. Dobrowolska, Phys. Rev. B 72, 155301 (2005).

${ }^{32}$ K. V. Kavokin, Phys. Status Solidi A 195, 592 (2003).

${ }^{33}$ M. E. Ware, E. A. Stinaff, D. Gammon, M. F. Doty, A. S. Bracker, D. Gershoni, V. L. Korenev, Ş. C. Bădescu, Y. LyandaGeller, and T. L. Reinecke, Phys. Rev. Lett. 95, 177403 (2005).

${ }^{34}$ An electromagnet $(|B| \leqslant 47 \mathrm{mT})$ was also used for the precise measurement in the low-field region and the same sharp linewidth was observed in the Hanle curve.

${ }^{35}$ The excitation power dependence is similar to that for the optical orientation of electrons in $n$-type semiconductors (Ref. 27).

${ }^{36}$ T. C. Damen, L. Viña, J. E. Cunningham, J. Shah, and L. J. Sham,
Phys. Rev. Lett. 67, 3432 (1991).

${ }^{37}$ M. Dyakonov, X. Marie, T. Amand, P. Le Jeune, D. Robart, M. Brousseau, and J. Barrau, Phys. Rev. B 56, 10412 (1997).

${ }^{38}$ Y. Masumoto, I. V. Ignatiev, K. Nishibayashi, T. Okuno, S. Yu. Verbin, and I. A. Yugova, J. Lumin. 108, 177 (2004).

${ }^{39}$ I. V. Ignatiev, T. Okuno, S. Yu. Verbin, I. A. Yugova, and Y. Masumoto, Physica E (Amsterdam) 17, 365 (2003).

${ }^{40}$ I. V. Ignatiev, I. E. Kozin, S. V. Nair, H.-W. Ren, S. Sugou, and Y. Masumoto, Phys. Rev. B 61, 15633 (2000).

${ }^{41}$ I. V. Ignatiev, I. E. Kozin, V. G. Davydov, S. V. Nair, J.-S. Lee, H.-W. Ren, S. Sugou, and Y. Masumoto, Phys. Rev. B 63, 075316 (2001).

${ }^{42}$ Semiconductor Quantum Dots-Physics, Spectroscopy and Applications, edited by Y. Masumoto and T. Takagahara (Springer, Berlin, 2002).

${ }^{43}$ The requirement $T_{2}^{*} \leqslant T_{2} \leqslant 2 T_{1}$ holds.

${ }^{44}$ I. A. Merkulov, Al. L. Efros, and M. Rosen, Phys. Rev. B 65, 205309 (2002)

${ }^{45}$ A. V. Khaetskii, D. Loss, and L. Glazman, Phys. Rev. Lett. 88, 186802 (2002).

${ }^{46}$ S. I. Erlingsson and Y. V. Nazarov, Phys. Rev. B 66, 155327 (2002).

${ }^{47}$ D. Gammon, Al. L. Efros, T. A. Kennedy, M. Rosen, D. S. Katzer, D. Park, S. W. Brown, V. L. Korenev, and I. A. Merkulov, Phys. Rev. Lett. 86, 5176 (2001).

${ }^{48}$ P.-F. Braun, X. Marie, L. Lombez, B. Urbaszek, T. Amand, P. Renucci, V. K. Kalevich, K. V. Kavokin, O. Krebs, P. Voisin, and Y. Masumoto, Phys. Rev. Lett. 94, 116601 (2005).

${ }^{49}$ W. Kohn, in Solid State Physics, edited by F. Seitz and D. Turnbull (Academic Press, New York, 1957), Vol. 5, p. 257.

${ }^{50}$ A. Abragam and B. Bleaney, Electron Paramagnetic Resonance of Transition Ions (Clarendon Press, Oxford, 1970). 